\title{
Left Ventricular Suction in Right Ventricular Dysfunction
}

\author{
Trainini Jorge $\mathrm{C}^{1 *}$, Trainini Alejandro ${ }^{2}$, Valle Cabezas Jesus ${ }^{3}$ and Cabo Javier ${ }^{4}$ \\ ${ }^{1}$ Universidad de Avellaneda, Argentina \\ ${ }^{2}$ Department of Cardiovascular Surgery, President Peron Hospital, Argentina \\ ${ }^{3}$ National Institute of Aerospace Technology, Sub directorate General of Naval Systems, Spain \\ ${ }^{4}$ Hospital NISA Pardo de Aravaca, Madrid, Spain
}

*Corresponding author: Jorge Carlos Trainini, Universidad de Avellaneda, Buenos Aires, Argentina

\begin{abstract}
Introduction and Objective: Recent research has determined that the "cardiac suction" phase occurs between systole and diastole. The aim of this work was to analyze the suction capacity of the left ventricle after excluding the right ventricle through an atriopulmonary bridge.

Methods: An atriopulmonary bridge was performed on six dogs, followed by right coronary artery occlusion to generate right ventricular dysfunction. Cardiac output (CO), cardiac index (CI), systolic index (SI), left ventricular stroke work index (LVSWI), right ventricular stroke work index (RVSWI), systemic vascular resistance (SVR) and pulmonary vascular resistance (PVR) were evaluated using a Swan Ganz catheter. Recordings were acquired at baseline before any procedure, 60 minutes after right coronary occlusion with the atriopulmonary bridge connection closed and 60 minutes after opening the atriopulmonary connection. At the end of the experiment, coronary angiography and histological examination were performed to verify the right coronary occlusion.

Results: Sixty minutes of coronary occlusion produced decreased CO (3.43 to $2.25 \mathrm{l} / \mathrm{min}$ ), CI (5.22 to $3.39 \mathrm{l} / \mathrm{m}^{2}$ ), SI (41.5 to 20.8 $\mathrm{ml} /$ beat $/ \mathrm{m} 2$ ), LVSWI (53.3 to $14.4 \mathrm{~g} \times \mathrm{m} / \mathrm{beat} / \mathrm{m}^{2}$ ), and RVSWI (1.61 to $-1.97 \mathrm{~g} \times \mathrm{m} / \mathrm{beat} / \mathrm{m}^{2}$ ). When the atriopulmonary bridge was opened, CO increased to $3.39 \mathrm{l} / \mathrm{min}(\mathrm{p}<0.05)$, CI to $4.95 \mathrm{l} / \mathrm{m}^{2}(\mathrm{p}<0.05)$, SI to $45.1 \mathrm{ml} / \mathrm{beat} / \mathrm{m}^{2}(\mathrm{p}<0.05)$, LVSWI to $40.8 \mathrm{~g} \times \mathrm{m} / \mathrm{beat} /$ $\mathrm{m}^{2}(\mathrm{p}<0.05)$ and RVSWI to $1.57 \mathrm{~g} \times \mathrm{m} / \mathrm{beat} / \mathrm{m}^{2}(\mathrm{p}<0.05)$, and right atrial pressure decreased from 10.6 to $3 \mathrm{mmHg}(\mathrm{p}<0.05)$ and PVR from 109 to $48.9 \mathrm{dyn} / \mathrm{s} / \mathrm{cm}^{-5}$ (ns).

Conclusion: As demonstrated by means of an atriopulmonary bridge, right ventricular dysfunction experimentally induced by ischemia is compensated by a left ventricular suction mechanism, restoring normal circulatory parameters.
\end{abstract}

Keywords: Ventricular suction; Atriopulmonary Bridge; Fontan; right ventricular exclusion; isovolumic diastolic phase.

Abbreviations: CI: Cardiac index; SI: Systolic index; LVSWI: Left ventricular stroke work index; RVSWI: Right ventricular stroke work index; SVR: Systemic vascular resistance; PVR: Pulmonary vascular resistance

\section{Introduction}

Recent studies have demonstrated an active suction phase between systole and diastole [1-4], with muscle contraction, energy expenditure and decreased intraventricular pressure. This active suction generates a gradient between peripheral and ventricular pressures, allowing blood flow into the heart [5-10]. Circulation is a dynamic process in which the heart, the vascular system and resistances modify their properties adapting according to the circulatory needs. From the point of view of blood oxygenation (though not histologically), we consider the systemic venous system, the right ventricle and the pulmonary artery as belonging to the venous system, while the pulmonary veins, the left ventricle and the arteries make up the arterial system. The systemic and pulmonary capillaries connect both systems, assuming that the filling pressures are similar in both sides (venous and arterial). Central venous pressure and pulmonary capillary pressure are comparable, the latter being the load the venous system offers 
to the left ventricle. The small difference between peripheral and heart pressure needs a suction energy in the left ventricle to generate intraventricular depression.

Although both ventricles manage similar volumes, their pressures are different. The left ventricular suction energy explains right ventricular filling. In the present study, we experimentally performed an atrioventricular bridge and generated right ventricular dysfunction, leaving the left ventricle as the only contractile component. In these conditions of right ventricular dysfunction, we analyzed whether the atriopulmonary bridge connection improved the hemodynamic conditions based on the left ventricular suction mechanism.

\section{Material and Methods}

The present study was conducted at Universidad de Avellaneda in Buenos Aires (Argentina), after approval by the institutional Ethics Committee. Six adult mongrel dogs slated for euthanasia, with mean weight of $17 \mathrm{~kg}$ and $0.72 \mathrm{~m}^{2}$ (range 0.56-0.96) body surface area, were used in the study. The experiments were performed under "Arrive" regulations and according to the United Kingdom 1996 Act on scientific procedures on animals, the EU Directive 2010/63/EU for experimental animals and the "National Institutes of Health" guide for the care and use of laboratory animals (NIH Publication No. 8023, updated in 1978). The animals were sedated with ketamine $(10 \mathrm{mg} / \mathrm{kg})$ and femoral artery and vein cannulation was performed for fluid infusion and arterial and central venous pressure monitoring. Ventilation was controlled with intermittent positive pressure using a Taka-Vent 550 respirator with $100 \%$ oxygen, airway flow of $9 \mathrm{l} / \mathrm{min}$ and positive pressure of $11 \mathrm{~cm} \mathrm{H}_{2} \mathrm{O}$. Anesthesia was maintained with $0.5-2 \%$ enflurane and fentanyl (5 $\mathrm{mg} / \mathrm{kg}$ ).

\section{Surgical Protocol}

Heparin (1 mg/kg) was administered after median sternotomy and pericardiectomy. The atriopulmonary bridge connection was performed between the right atrial appendage and the pulmonary artery (through partial clamping) with an $8 \mathrm{~mm}$ diameter woven conduit. A Swan-Ganz catheter was inserted into the pulmonary artery, connected to a transducer and two recording channels, and a second catheter was placed in the right atrium. Cardiac output was assessed using the thermodilution technique.

Variables Recorded: Right atrial pressure, pulmonary artery pressure, pulmonary capillary pressure, mean arterial pressure, heart rate and cardiac output were recorded.

\section{Calculations and formulas:}

Cardiac index (CI): cardiac output/body surface area $=1 / \mathrm{min} / \mathrm{m}^{2}$

Systolic index (SI): CI/heart rate $=\mathrm{ml} / \mathrm{beat} / \mathrm{m}^{2}$

Left ventricular stroke work index (LVSWI): (mean arterial pressure-pulmonary capillary pressure) $\times \mathrm{SI} \times 0.0136=\mathrm{g} \times \mathrm{m} /$ beat $/ \mathrm{m}^{2}$
Right ventricular stroke work index (RVSWI): (pulmonary arterial pressure-right atrial pressure) $\times$ SI $\times 0.0136=\mathrm{g} \times \mathrm{m} /$ beat $/ \mathrm{m}^{2}$

Systemic vascular resistance (SVR): (pulmonary arterial pressure-right atrial pressure)/cardiac output $\times 80=$ dyn $/ \mathrm{s} /$ $\mathrm{cm}^{-5}$

Pulmonary vascular resistance (PVR): (pulmonary arterial pressure- pulmonary capillary pressure/cardiac output $\times$ $80=$ dyn $/ \mathrm{s} / \mathrm{cm}^{-5}$

Measurements were taken at baseline before any procedure. Following right coronary occlusion by ligation at its origin, volume overload with saline was achieved to a threefold increase of right atrial pressure. After 60-minute ischemia, the atriopulmonary bridge was opened and kept patent during another 60 minutes. Hematocrit, $\mathrm{pH}, \mathrm{pO}_{2}$ and $\mathrm{pCO}_{2}$ were controlled at all times. The experiment was concluded after 120 minute-ischemia and the hearts were explanted. A coronary angiography was performed to verify the right coronary artery occlusion, and tissue samples were taken and fixed in 10\% formalin for histological analysis. The right ventricle was sectioned, taking samples at different heights of the wall and additional samples near the left apex. Samples were processed in an autothecnicon tissue processor and stained with hematoxylin-eosin, periodic-acid Schiff (PAS) for glycogen and basic fuchsin dye for necrosis-ischemia.

\section{Statistical Analysis}

Analysis of variance for randomized block design, Tukey test for multiple comparisons and Friedman's test for non-parametric and post-hoc comparisons were used to analyze the data. Differences with $\mathrm{p}<0.05$ were considered as significant.

\section{Results}

Table 1 shows hemodynamic values at baseline, at 60 minutes of right coronary artery occlusion and at 60 minutes after atriopulmonary bridge connection. Both the right and left ventricles showed no changes in myofibrillar architecture, nucleus or transverse striation with hematoxylin-eosin staining. The PAS technique evidenced marked glycogen reduction in right ventricular sections, expressed as complete lack of magentacolored granules, almost throughout the entire wall thickness. Only a thin band of subepicardial parallel fibers and other isolated fibers in the papillary muscles presented scarce glycogen granules. Conversely, the left ventricle showed visible glycogen content. The almost massive loss of intracellular glycogen demonstrated by the PAS technique is due to the early change (first 30 minutes) observed in experimental animals with coronary occlusion.

\section{Discussion}

As confirmed by different studies, acute occlusion of the right coronary artery produces right ventricular dysfunction [11]. Table 1 reflects the decrease of RVSWI (1.61 to $-1.97 \mathrm{~g} \times \mathrm{m} / \mathrm{beat} / \mathrm{m}^{2}$ ) 
and of cardiac output (3.43 to $2.25 \mathrm{l} / \mathrm{min}$ ) following right coronary artery occlusion. The right ventricle expanded, increasing its atrial pressure and decreasing pulmonary capillary pressure. At autopsy, right ventricular dilation is frequent after its acute infarction. Moreover, PVR increased from 49.9 to $109 \mathrm{dyn} / \mathrm{s} / \mathrm{cm}^{-5}$ and the SI decreased from 41.5 to $20.8 \mathrm{ml} / \mathrm{beat} / \mathrm{m}^{2}$. The choice of 60 minutes between coronary occlusion and atriopulmonary bridge opening was not arbitrary. Previous works have established that a 40-minute period of regional ischemia produces irreversible ventricular injury
[11]. In our case, histological analysis confirmed right ventricular ischemia. It was seen that the injured right ventricle was not able to behave as a simple conduit vessel, as volume overload worsened its distention and impaired the SI (Table 1). All the animals evidenced right ventricular damage, with contractile dysfunction and lack of synchronization between both ventricles. Our model eliminated the pericardial protective function, which in case of being intact would have attenuated right ventricular dilation secondary to ischemia.

Table 1: Hemodynamic values.

\begin{tabular}{|c|c|c|c|c|}
\hline & Basal & 60' RCO & 60' RA-PA & p value \\
\hline RAP (mmHg) & $3.66 \pm 1.22$ & $10.16 \pm 3.06$ & $3.00 \pm 1.27$ & $\mathrm{p}<0.05$ \\
\hline PAP (mmHg) & $6.41 \pm 3.29$ & $3.41 \pm 1.43$ & $6.25 \pm 2.56$ & $\mathrm{p}<0.05$ \\
\hline PCP (mmHg) & $5.00 \pm 3.41$ & $1.66 \pm 2.25$ & $4.83 \pm 2.64$ & NS \\
\hline MAP (mmHg) & $91.60 \pm 14.38$ & $51.00 \pm 9.38$ & $72.50 \pm 9.87$ & $\mathrm{p}<0.05$ \\
\hline HR (beat/m) & $121 \pm 7.99$ & $153 \pm 25.00$ & $115 \pm 13.55$ & $\mathrm{p}<0.05$ \\
\hline $\mathrm{CO}(\mathrm{l} / \mathrm{m})$ & $3.43 \pm 1.83$ & $2.25 \pm 1.04$ & $3.29 \pm 1.66$ & $\mathrm{p}<0.05$ \\
\hline $\mathrm{CI}\left(\mathrm{l} / \mathrm{m}^{2}\right)$ & $5.22 \pm 3.72$ & $3.39 \pm 2.08$ & $4.95 \pm 3.36$ & $\mathrm{p}<0.05$ \\
\hline LVSWI $\left(\mathrm{g} \times \mathrm{m} /\right.$ beat $\left./ \mathrm{m}^{2}\right)$ & $53.30 \pm 44.31$ & $14.40 \pm 7.34$ & $40.80 \pm 31.61$ & $\mathrm{p}<0.05$ \\
\hline RVSWI ( $\mathrm{g} \times \mathrm{m} /$ beat $/ \mathrm{m}^{2}$ ) & $1.61 \pm 0.92$ & $-1.97 \pm 1.76$ & $1.57 \pm 0.67$ & $\mathrm{p}<0.05$ \\
\hline SVR (dyn $/ \mathrm{s} / \mathrm{cm}^{-5}$ ) & $2.615 \pm 1.581$ & $1.605 \pm 411.2$ & $2.356 \pm 1.800$ & NS \\
\hline PVR (dyn/s/cm ${ }^{-5}$ ) & $49.90 \pm 29.18$ & $109 \pm 77.81$ & $48.9 \pm 21.05$ & NS \\
\hline SI (ml/beat/m²) & $41.50 \pm 26.38$ & $20.80 \pm 8.97$ & $45.10 \pm 34.93$ & $\mathrm{p}<0.05$ \\
\hline
\end{tabular}

RCO: right coronary occlusion; RA-PA: right atrium-pulmonary artery bridge; RAP: right atrial pressure; PAP: pulmonary arterial pressure; PCP: pulmonary capillary pressure; MAP: mean arterial pressure; HR: heart rate; CO: cardiac output; CI: cardiac index; LVSWI: left ventricular stroke work index; RVSWI: right ventricular stroke work index; SVR: systemic vascular resistance; PVR: pulmonary vascular resistance; SI: systolic index.

In view of the expected post-ischemic right ventricular dilation (worsening after increasing preload) we applied Fontan's principle: right atrial connection to the pulmonary artery, avoiding the dysfunctional right ventricle [12]. We sought to increase left ventricular preload and relieve right ventricular distention. Right ventricular ischemia reduced cardiac output to $65 \%$ and LVSWI to $27 \%$ their baseline values. Opening of the atriopulmonary bridge connection after 60-minute ischemia produced hemodynamic recovery by unloading the right atrium into the pulmonary artery. Cardiac output improved to $95 \%$ its baseline value, recovering to $3.29 \mathrm{l} / \mathrm{min}(\mathrm{p}<0.05)$, SI to $45.1 \mathrm{ml} / \mathrm{beat} / \mathrm{m}^{2}(\mathrm{p}<0.05)$, LVSWI to $40.8 \mathrm{~g} \times \mathrm{m} /$ beat $/ \mathrm{m}^{2}(\mathrm{p}<0.05)$ and RVSWI to $1.57 \mathrm{~g} \times \mathrm{m} / \mathrm{beat} / \mathrm{m}^{2}$ $(\mathrm{p}<0.05)$. Also, right ventricular pressure decreased from 10.16 to $3 \mathrm{mmHg}(\mathrm{p}<0.05)$ and PVR from 109 to $48.9 \mathrm{dyn} / \mathrm{s} / \mathrm{cm}^{-5}$ (ns). The new scenario that emerges after opening the atriopulmonary bridge connection by eliminating the passage through the dysfunctional right ventricle, transfers the right circulatory mechanism exclusively to the left ventricular suction capacity. Our experimental model shows how the obstacle of a dysfunctional right ventricle can be overcome bypassing this chamber with an atriopulmonary bridge, with the sole prior condition of acceptable pulmonary resistances. Thus, the left ventricular suction mechanism acts as a cardiac flow engine.
The increase in SI was higher than expected, considering that the pressure difference between the right atrium and the pulmonary artery disappeared rapidly when the atriopulmonary bridge connection was opened. It is possible that reduced right preload conditions a slight improvement of the right ventricle by not worsening the ischemic effects, thus contributing to the increase in SI after a discrete functional recovery. This analysis is concurrent with previous investigations [1-5] where the isovolumic diastolic phase was studied as an active phenomenon generated by a myocardial contraction that tends to expand the left ventricular apex-base distance after the ejective phase, producing a suction effect similar to that of a "plunger". A drop in intraventricular pressure is generated that, in turn, elicits ventricular suction. It is an active process during the isovolumic phase, which is erroneously considered as diastolic. When this pressure is sufficiently negative $(-10 \mathrm{mmHg})$ and the left ventricle is elongated and "uncoiled", the mitral valve opens with the ensuing rapid blood filling from the atrium. This suction phase between systole and diastole of the human cardiac cycle lasts between 100 and 120 ms with active muscle contraction and a drop of intraventricular pressure below zero, as shown by Tyberg [13] with balloon mitral valve occlusion in the dog. Fontan procedures, with atriopulmonary bypass that avoid the right ventricle, clinically show the efficiency of the left 
ventricular suction mechanism. Its validity is confirmed even before recent physio mechanical investigations. The same consideration should be applied to left circulatory mechanics, where blood is bypassed from the left ventricle into the aorta. It can be concluded that suction due to the elastic recoil of the helical ventricular structure is an active process. Myocardial contraction occurs during the left ventricular isovolumic phase. In cases of ventricular dilation, this suction mechanism ("plunger") becomes more precarious. This concept may help to establish a new evaluation of heart failure and its clinical severity [14-16].

\section{Conclusion}

As demonstrated using an atriopulmonary bridge, right ventricular dysfunction produced experimentally by ischemia is compensated by a left ventricular suction mechanism, maintaining normal circulatory parameters

\section{Appendix}

Cardiac output (CO) is given by:

$$
C O=\left(E^{1}+E^{2}\right) /\left(R^{1}+R^{2}\right)
$$

where E1: left ventricular energy; $\mathrm{E}^{2}$ : right ventricular energy; $\mathrm{R}^{1}$ : systemic vascular resistance; and $\mathrm{R}^{2}$ : pulmonary vascular resistance.

$E^{1} / R^{1}=E^{2} / R^{2}$ shows the output energy and vascular resistance ratio between both circulatory systems; then:

$$
C O=E^{1} / R^{1}=E^{2} / R^{2} ; y Q^{1}=Q^{2}
$$

With $\mathrm{Q}^{1}=$ left ventricular flow potential; $\mathrm{Q}^{2}=$ = right ventricular flow potential

Venous pressure is the same in both circulatory systems. If the right ventricle is withdrawn from the circuit $\left(E^{2}=0\right)$, cardiac energy after surgery results as:

$$
C O=E^{1}\left(R^{1}+R^{2}\right)
$$

\section{References}

1. Trainini JC, Elencwajg B, López Cabanillas N, Herreros J, Lago N, et al (2015) Electrical Propagation in the Mechanisms of Torsion and Suction in a Three-phase. Rev Argent Cardiol 83: 416-423.
2. Trainini JC, Elencwajg B, López Cabanillas N, Herreros J, Lago N, et al. (2015) Basis of the New Cardiac Mechaniscs The Suction Pump. Ed Lumen Bs, pp. 112.

3. Torrent Guasp F (1998) Structure and function of the heart. Rev Esp Cardiol 51: 91-102.

4. Brecher GA (1956) Experimental evidence of ventricular diastolic function. Circ Res 4(5): 513-518.

5. Torrent Guasp F, Kocika MJ, Corno A, Masashi K, Cox J, et al. (2004) Systolic ventricular filling. Eur J Cardio-thorac Surg 25(3): 376-86.

6. Sengupta PP, Krishnamoorthy VK, Korinek J, Narula J, Vannan MA, et al. (2007) Left ventricular form and function revisited: applied translational science to cardiovascular ultrasound imaging. J Am Soc Echocardiogr 20(5): 539-551.

7. Carreras F, Ballester M, Pujadas S, Leta R, Pons Lladó G (2006) Morphological and functional evidences of the helical heart from noninvasive cardiac imaging. Eur J Cardiothoracic Surg 29(Suppl 1): S50-S55.

8. Cosín Aguilar J, Hernándiz Martínez A (2013) The disposition of the myocardial fibers in a band conditions the morphology and function of the heart. Rev Esp Cardiol 66: 768-770.

9. Poveda F, Gil D, MartíE, Andaluz A, Ballester M, et al. (2013) Tractographic study of the helical anatomy of the ventricular myocardium by means of magnetic resonance by diffusion tensor. Rev Esp Cardiol 66: 782-790.

10. Wu MT, Tseng WYI, Su MYM, Liu ChP, Chiou KR, et al. (2006) Diffusion tensor magnetic resonance imaging mapping the fiber architecture remodeling in human myocardium after infarction. Correlation with viability and wall motion. Circulation 114: 1036-1045.

11. Jennings RB, Reimer KA (1983) Factors involved in salvaging ischemic myocardium. Effect of reperfusion of arterial blood. Circulation 68(Suppl I): 25-36.

12. Fontan F, Baudet E (1971) Surgical repair of tricuspid atresia. Thorax 26(3): 240-248.

13. Tyberg JV, Keon WJ, Sonnenblick EH, Urschel J (1970) Mechanics of ventricular disease. Cardiovasc Res 4: 423-428.

14. Trainini JC, Elencwajg B, López Cabanillas N, Herreros J, Lago N (2015) Electrophysiological Bases of Torsión and Suction in the Continuous Cardiac Band Model. Anat Physiol 5: S4.

15. Trainini JC, Herreros J, Cabo J, Otero Coto E, Cosín Aguilar J (2011) The cardiac suction pump. Application of the myocardial band of Torrent Guasp to the surgical treatment of heart failure. Cir Cardiovasc 18(2): 103-112.

16. Weber KT, Janicki JS, Shroff SG, Likoff MJ, Sutton MJ (1983) The right ventricle: physiologic and pathophysiologic considerations. Crit Care Med 11(5): 323-328.

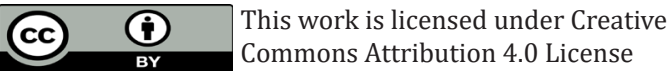

To Submit Your Article Click Here:

Submit Article

\section{ACRR}

DOI: 10.32474/ACR.2019.02.000127

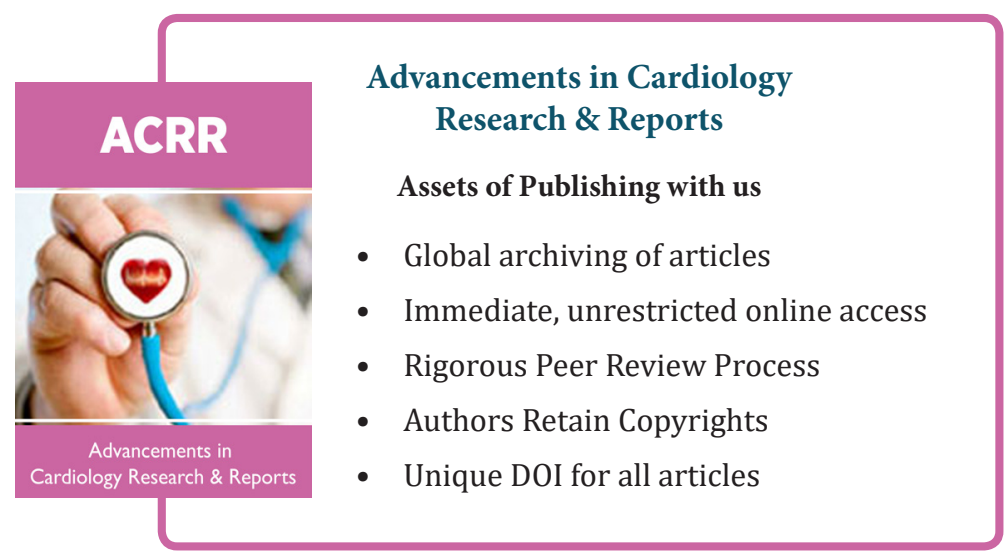

